\title{
Manipulation of Stem Cells and their Microenvironment for Tissue Engineering
}

\author{
Adrian McArdle ${ }^{1}$, Kevin J. Paik ${ }^{1}$, Michael T. Chung ${ }^{1}$, Michael S. Hu¹, Graham Walmsley ${ }^{1}$, Andrew Zimmermann ${ }^{1}$, Victor W. Wong ${ }^{1}$, Brian \\ Hsueh ${ }^{1}$, Andrew S. Chung ${ }^{1}$, Geoffrey C. Gurtner ${ }^{1}$, H. Peter Lorenz ${ }^{1}$, Michael T. Longaker ${ }^{1,2}$ and Derrick C. Wan ${ }^{1,3^{*}}$
}

${ }^{1}$ Hagey Laboratory for Pediatric Regenerative Medicine, Department of Surgery, Plastic and Reconstructive Surgery Division, Stanford University School of Medicine, Stanford, California, USA

${ }^{2}$ Institute for Stem Cell Biology and Regenerative Medicine, Stanford University, Stanford, California, USA

${ }^{3}$ Hagey Laboratory for Pediatric Regenerative Medicine, Stanford University School of Medicine, Stanford University, Stanford, California, USA

\begin{abstract}
The potential for stem cells to serve as cellular building blocks for reconstruction of complex defects has generated huge enthusiasm in the field of regenerative medicine. Skeletal and soft tissue defects due to trauma, tumor resection, and congenital anomalies present significant challenges for surgical reconstruction. The limitations of current treatment options have shifted attention to regenerative medicine, which has the potential to dramatically improve our ability to repair the human body. The possibility of regenerating lost or damaged tissues offers significant hope in the field of surgery. The present review article highlights recent progress in tissue-specific engineering with stem cells, and future directions for this rapidly evolving area of medicine.
\end{abstract}

Keywords: Stem cells; Tissue engineering; Niche; Microenvironment; Scaffold; Mesenchymal stem cells; Adipose-derived stem cells; Induced pluripotent stem cells; Plastic surgery

\section{Introduction}

Regeneration of damaged adult tissues requires the existence of cells capable of proliferation and differentiation that will contribute functionally to the reparative process of a tissue. In a wide variety of conditions, ranging from osteoarthritis to cerebrovascular accidents, the intrinsic ability of the body to repair itself is insufficient. The field of regenerative medicine and tissue engineering holds promise in treating these conditions, especially with the renewed impetus that has arisen from the discovery of stem cells. The addition of stem cells to our regenerative medicine armamentarium has opened up new avenues with the potential for developing stem cell-based therapies for the treatment of these conditions.

Stem cells possess a distinct ability to self-renew and differentiate, making them a more attractive candidate cell for cell-based therapies when compared with other cell types like somatic cells. Stem cells encompass a large class of cell types, ranging from pluripotent embryonic stem cells (ESCs) and induced pluripotent stem cells (iPSCs) to fate-restricted, multipotent adult stem cells such as BMMSCs and ASCs. Ethical concerns have limited the use of embryonic stem cells in regenerative medicine, and current focus is on induced pluripotent stem cells (iPSCs) and adult stem cells. Different putative sources of adult stem cells have been identified, from sources such as bone marrow, adipose tissue and umbilical cord blood, each offering their distinct advantages and disadvantages.

Adult stem cells are an exciting cell source for regenerative medicine, owing mostly to their relative ease of harvest and the ability to yield large quantities of cells. In addition, most of the tissues that require reconstruction in surgery, like bone and muscle, are mesenchymal in origin and are therefore a logical cell type for use in regenerative surgery. iPSCs are the most recent discovery which offers huge potential for clinical translation.

To harness the full potential of a particular cell type in regenerative medicine, a clear understanding of the contribution from the specialized microenvironment that governs stem cell behavior is key to manipulating these cells to generate robust, clinically relevant, engineered tissues. Understanding the complex interplay of the stem cells and their specialized microenvironment, known as the niche, will provide key strategies for the biomimetic stimulation of both implanted and native stem cells, and this avenue is being actively pursued in regenerative medicine. Study of niche microenvironments remains in their infancy and this area of study are emerging as a key field within stem cell biology.

Whilst a lot of study in stem cell biology has focused on finding the ideal cell source for tissue engineering, it is clear that in order to fully exploit the true potential of these cells in a clinical environment; we must optimize the delivery system for these cells. The cellular component is only one facet of the niche, and the cellular component which consists of the biomimetic substrate and inductive cues, is equally as important in dictating the regenerative process in vivo. In order to manipulate these signals, the field of tissue engineering has employed a combination of biological and material engineering approaches. Here we will discuss the current research surrounding the investigation of the core components of tissue regeneration - the cells, the biomimetic substrate and the inductive cues. Based on our experience we will draw on particular reference to surgical applications.

\section{Skeletal Tissue}

The treatment of bone degenerative diseases and congenital craniofacial disorders stands to benefit greatly from the development of reliable, efficient, and safe regenerative therapies. More than 400 publications have investigated bone tissue engineering in rodents to date. However, few studies have translated this approach to larger animals such as sheep, goats, and dogs [1-3]. From a clinical perspective, bone tissue engineering holds great promise but has thus

*Corresponding author: Derrick C. Wan, Hagey Laboratory for Pediatric Regenerative Medicine, Stanford University School of Medicine, 257 Campus Drive, Stanford University, Stanford, CA 94305-5148, USA, Tel: (650)723-7073; Fax: (650) 736-1705; E-mail: dwan@stanford.edu

Received May 30, 2013; Accepted June 18, 2013; Published June 25, 2013

Citation: McArdle A, Paik KJ, Chung MT, Hu MS, Walmsley G, et al. (2013) Manipulation of Stem Cells and their Microenvironment for Tissue Engineering Surgery Curr Res 3: 134. doi:10.4172/2161-1076.1000134

Copyright: (c) 2013 McArdle A, et al. This is an open-access article distributed under the terms of the Creative Commons Attribution License, which permits unrestricted use, distribution, and reproduction in any medium, provided the original author and source are credited. 
far failed to deliver significant results to the bedside. Only a handful of studies with successful reconstructions have been published to date in humans $[4,5]$.

\section{The seed: cell source}

Mesenchymal stem cells have demonstrated therapeutic value for the regeneration of musculoskeletal tissue due to their ability to selfrenew and differentiate along all mesenchymal lineages [6]. Indeed, the tri-lineage potential of MSCs to differentiate into adipocytic, chondrocytic, and osteocytic lineages in response to extracellular signals is remarkable. Perhaps most importantly for translational medicine, MSCs can be readily harvested from bone marrow aspirate, blood, and adipose tissue making them an ideal autologous cell source. MSCs represent a heterogeneous population of cells largely due to isolation techniques that rely upon cell culture adherence or density-gradient fractionation. A MSC surface marker profile including expression for CD73, CD106, CD146, and STRO-1 and absence of CD11b, CD31, CD34, CD45, and CD117 currently exists, but no single definitive marker has been identified to date [7]. This lack of a definitive marker has made in vivo lineage tracing and niche investigation challenging (Figure 1).

As the major source of osteoblasts and osteoprogenitors, MSCs exert significant control over the process of bone formation during physiologic and pathologic states. Adjusting the quantity, localization, and differentiation potential of MSCs thus provides a direct means to modulate therapeutic bone regeneration. The "trophic" action of MSCs as producers of a wide spectrum of growth factors with antiinflammatory, anti-apoptotic, proliferative, and pro-angiogenic properties has also been shown to enhance osteogenesis as well $[8,9]$. In the context of transplantation, MSCs are thought to act as signaling centers that orchestrate osteogenic differentiation along with resident host cells. Growth factors (Bone Morphogenetic Protein (BMP)-2, Fibroblast Growth Factor (FGF)-2), gene delivery (Runx2, WISP-1), and synthetic biomimetic scaffolds (polyglycolic acid, polylactic acid) have all been shown to amplify these osteogenic properties of MSCs [10-12]. Additionally, tissue culture conditions such as low oxygen tension and cell seeding density increase the osteogenic activity and longevity of cultured MSCs; however, much controversy remains

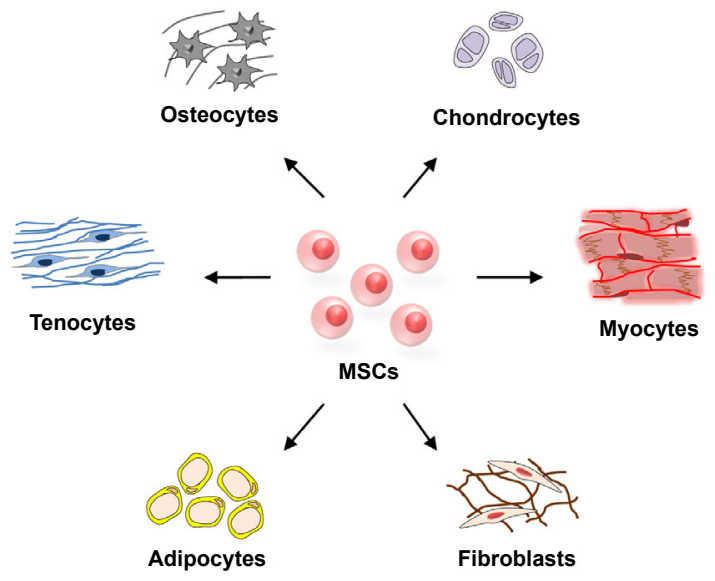

Figure 1: Mesenchymal stem cells-such as bone marrow-derived mesenchymal stem cells and adipose-derived stem cells-have the potential to differentiate into various lineages, making them invaluable tools in tissue engineering. regarding optimal expansion conditions [13-15]. Optimizing these conditions will likely depend on the therapeutic application in question (Figure 5).

The discovery of iPSCs by Yamanaka et al. opened another promising avenue of exploration for cell-based bone regeneration strategies [16]. Both iPSCs and ESCs are capable of differentiation into numerous cell types from each of the three germ layers. Adaptation of existing ESC differentiation protocols has allowed for the osteogenic differentiation of iPSCs $[17,18]$ and more recently, generation of osteoblasts from iPSCs was achieved via Runx 2 adenoviral transduction [19]. Li et al. [20] reported the derivation of MSC-like cells from mouse iPSCs. The MSC-like cells expressed putative MSC markers, deposited calcium in vitro, and differentiated into adipocytes or osteoblasts upon exposure to appropriate culture conditions. Furthermore, Lian et al. demonstrated the therapeutic potential of deriving MSCs from iPSCs in a mouse limb ischemia transplantation model [21].

Recent investigations have convincingly demonstrated the osteogenic potential of iPSCs in vivo. Many studies have either transplanted pre-differentiated iPSCs or iPSC-seeded scaffolds into ectopic sites (subcutaneous or sub-renal capsule) of immunodeficient mice and assessed bone formation. However, most studies have relied upon ex vivo iPSC differentiation prior to transplantation. Levi and colleagues demonstrated successful in vivo differentiation by seeding iPSCs overnight onto a Bone Morphogenetic Protein 2-releasing polyL-lactic acid scaffold to heal critical-sized mouse calvarial defects [22]. In addition, the utility of iPSCs for bone engineering has been shown across many orthotopic sites. Duan et al. highlighted the value of iPSCs for periodontal tissue engineering in a study showing that iPSCs combined with enamel matrix derivatives promoted the formation of new cementum, alveolar bone, and periodontal ligament [23].

\section{The soil: skeletal stem cell niche}

Natural biomaterials such as collagen, gelatin, chitosan, hyaluronan, and chondroitin sulfate in combination with cell and growth factorbased approaches have been used in the regeneration of bone, as well as cartilage, ligament, tendon, skin, and adipose tissue [24-28]. These materials offer high biocompatibility and the ability to replicate specific characteristics of native extracellular matrix thereby enhancing cell migration, proliferation, differentiation, and matrix deposition. However, disadvantages associated with natural biomaterial scaffolds include the potential for pathogen contamination during preparation from animal sources and laborious purification protocols.

More recently synthetic scaffolds composed of alpha-hydroxy ester polymers and ceramics have been used successfully for controlled in vivo and ex vivo bone regeneration [29,30]. Synthetic biomaterials offer high stability and low variation in physicochemical properties. Poly (alpha-hydroxy esters) such as polyglycolic acid and polylactic acid are biodegradable through ester bond hydrolysis into non-toxic glycolic and lactic acid respectively. Depending upon the application in question, polymer degradation rates can be modulated by altering co-polymer ratios, molecular weight, and crystallinity [31]. Techniques such as gas-foaming, sintering, and crystal leaching allow for the production of three-dimensional scaffolds with specific micro and macro-architectures, biomechanical properties, and pore sizes. More recently, electro spinning has come to the forefront as a highly efficient technique for the fabrication of polymer nano fibers. However, despite significant advantages, improving the biocompatibility and biomechanical properties of polymeric scaffolds are key obstacles. Ceramic scaffolds include calcium sulfates, calcium phosphates, and bioactive glasses. As a major component of bone, calcium phosphate 
based scaffolds such as hydroxyapatite and beta-tricalcium phosphate have been studied extensively [3,32]. These materials possess high compressive strength, variable degradation rates, and multi-scale porosity. Combinations of micro and macro-porosity produce both lamellar and woven bone formation while non-microporous scaffolds do not [33]. Recently, silica and zinc oxide doping has allowed for variations in the biomechanical properties of calcium phosphate based ceramic scaffolds [34]. Improving the reproducibility of ceramic scaffold manufacturing remains a challenge.

\section{Surgical applications for bone tissue engineering}

Autologous bone grafting remains the gold standard for reconstruction of osseous defects. However, autologous bone grafting is limited by donor site complications such as chronic pain, bleeding, and infection. Bone tissue engineering offers a means to decrease the amount of harvested bone required for reconstruction, and may thus help to minimize or eliminate donor site morbidity. Critical to the advancement of bone tissue engineering in the context of human disease is the incorporation of techniques for the induction of axial vascularization rather than relying-as many studies on model organisms have done-upon extrinsic neovascularization (Figure 2).

Optimizing cell and scaffold-based bone tissue engineering in the context of favorable vascular axes and vascularized flaps is essential for translation to the clinical setting. Some progress has been made on this front. Collagen type I scaffolds seeded with osteoprogenitors derived from cultured bone marrow stromal cells have been used to fabricate pedicled bone flaps via the carotid artery in mice [35]. Prefabricated vascularized bone grafts have also been employed in the clinical setting for mandibular reconstruction. Warnke and colleagues [36] used a custom-made titanium cage filled with bone marrow aspirate, OP-1, and xenogenic bone minerals to successfully repair a large mandibular defect. Despite these reports though, more studies on bone engineering with a focus on integrating clinically viable vascularization strategies are still needed before we see a significant impact at the bedside.

\section{Adipose Tissue}

Reconstruction of soft tissue defects resulting from injury, oncological resection, congenital anomalies, and aging can result in impaired aesthetic appearance, function, and the psychological well being of patients. Repair of these soft tissue defects often requires restoration of missing volume with a natural, synthetic, or hybrid material to enhance aesthetic contour. The ideal material for soft tissue reconstruction should look and feel natural, be stable after implantation

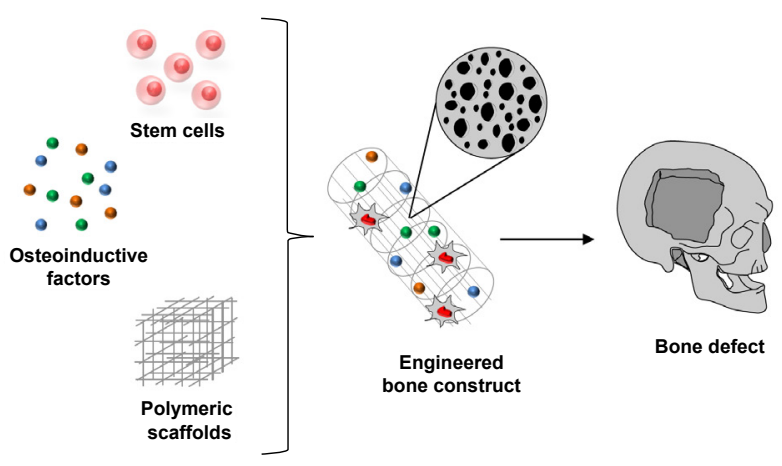

Figure 2: In bone tissue engineering, MSCs may be combined with growth factors such as BMP-2 and a pro-osteogenic scaffold microniche to promote enhanced healing of skeletal defects. or injection, and be fully integrated or replaced by host tissue over time. Numerous substances have been used for the reconstruction and/ or augmentation of soft tissues, but presently available filler materials present significant drawbacks to their use. Autologous soft-tissue grafts are therefore favored, as allografts, xenograft, and synthetic materials can be associated with immune rejection, allergic reaction, implant migration or resorption, and failure to integrate into host tissues [37, 38].

When compared to other soft tissue fillers, autologous fat exhibits many qualities of the ideal filler. It is biocompatible, readily available, easy to harvest, and naturally integrated into the host tissues. However, only small defects can be corrected with injected autologous fat, and free fat transfer often yields varying degrees of resorption, requiring repeated treatments to maintain the desired volume. For larger volumes, autologous tissue transfer using vascularized free flaps may be employed, but there are high costs in terms of donor site morbidity and deformity. Alternatively, tissue engineering strategies have the potential to overcome many deficiencies associated with autologous fat grafting and injection of synthetic materials [39-43]. Importantly, development of an adipose tissue regenerative approach requires coordination between all key aspects of tissue engineering including selection of an optimal cell source, scaffold material, and cellular environment.

\section{The seed: cell source}

There are two types of cellular building blocks typically employed for adipose tissue engineering. These include preadipocyte cell lines or primary cells. Preadipocytes possess high proliferative potential and the capacity to differentiate into adipose tissue. Although many adipogenic cell lines (3T3-F442a, Ob17) have been investigated, the classic cell type to study adipogenesis is the 3T3-L1 cell line, which was developed through clonal expansion of rodent-derived cells [4447]. While these cell lines circumvent many of the problems associated with primary cells, namely slower expansion and lower tolerance to cryopreservation, the differentiation capacity of primary cells, such as MSCs, may be more representative of true in vivo cellular behavior. Adult MSCs can self-replicate and also undergo adipogenic differentiation in response to appropriate signaling cues [6,48]. von Heimburg et al. [49] succeeded in engineering adipose tissue fusing collagen scaffolds combined with human ASCs. Importantly, freshly isolated stromal vascular fraction from adipose tissue contains a mixture of cells, which not only includes ASCs, but also endothelial cells, smooth muscle cells, pericytes, fibroblasts, and other circulating cells [50]. One explanation for variable success rates reported for in vivo adipogenic differentiation may be the different functional properties of these heterogeneous cell populations. Therefore, recent studies have sought to purify isolated subpopulations with the greatest potential for adipogenic differentiation using fluorescence-activated cell sorting. $\mathrm{Li}$ et al. [51] found that the "preadipocyte" population (CD31-/CD34+) showed the most adipogenic potential and demonstrated the highest expression of peroxisome proliferator-activated receptor- $\gamma$ after exposure to adipogenic differentiation medium. As studies continue to refine approaches to isolate an optimal cell for adipose tissue engineering strategies, ultimate translation to the clinical setting becomes increasingly tangible.

\section{The soil: adipose stem cell niche}

Another strategy to induce in vivo formation of adipose tissue is based on precursor stem cells already existing in the body without the need for exogenous transplantation of cells. This strategy revolves around niche-directed regulation of stem cell differentiation 
into adipocytes. Kawaguchi et al. [52] demonstrated that de novo adipogenesis in the mouse subcutis could be achieved by simply injecting a mixture of FGF-2 and Matrigel. Other attempts using these principles have included co-injection of gelatin microspheres with FGF-2 or insulin and insulin-like growth factor-1 [53-55]. Several other factors, including dexamethasone, thyroid hormone, epidermal growth factor, transforming growth factor- $\beta$, and platelet-derived growth factor have been shown to positively influence the rate of adipogenic differentiation in vitro and may potentially aid in development of a viable engineered adipose tissue construct in vivo [56-58] (Figure 3).

To develop an effective tissue engineering strategy, it is important to understand the complex physiology of adipose tissue and the signaling cascades that regulate adipogenesis. Adipose tissue is organized into a 3D tissue composed or ASCs and mature adipocytes in addition to stromal-vascular cells (interstitial cells, endothelial cells, pericytes). A complex micro vascular system entwined within a highly organized extracellular matrix (ECM) composed of collagen types I, III, IV, V, and VI and other ECM proteins is also present [59]. Advances in tissue engineering have demonstrated the feasibility of using $3 \mathrm{D}$ scaffolds to direct adipogenic differentiation in vivo. Both synthetic and natural polymers have been utilized extensively for this purpose. Traditional cell-based adipose tissue engineering strategies have used scaffolds for seeding cells to increase cellular adhesion. The concept is that as the scaffold degrades, transplanted cells would grow, proliferate, and eventually mature into adipose tissue. Patrick et al. [60] demonstrated the differentiation of ASCs seeded on polylactic co-glycolic acid scaffolds into mature adipocytes using this method.

Other cell-based adipose tissue engineering strategies have used hydrogels designed for restoration of tissue volume. These cellencapsulating hydrogels could be injected into soft tissue defects and would ideally restore the aesthetic contours by imparting a soft, smooth feel closely resembling that of natural tissue. Kim et al. demonstrated the feasibility of adipose tissue engineering using injectable, degradable alginate hydrogels with preconditioned human ASCs [61]. Newly generated tissues were semi-transparent and soft, grossly resembling adipose tissue.

\section{Surgical applications for adipose tissue engineering}

To date, the principle of engineering adipose tissue has been well demonstrated. Although autologous fat grafts are currently the best method of soft tissue augmentation that can be performed with minimal scarring and without complications associated with foreign materials, this strategy has shown unpredictable resorption rates

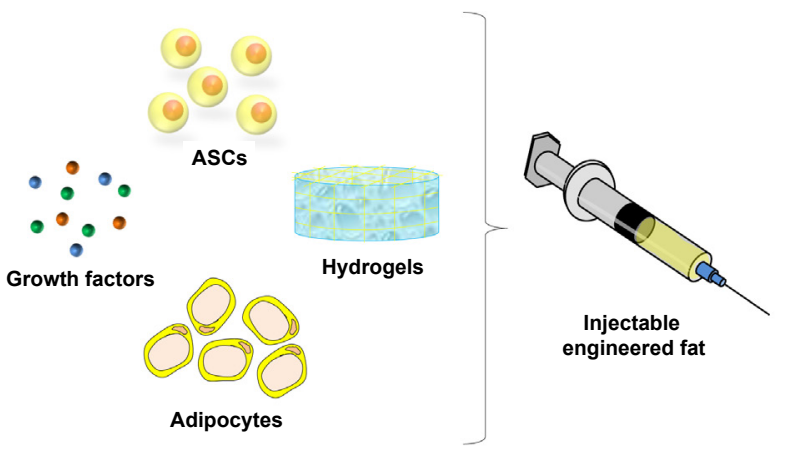

Figure 3: In adipose tissue engineering, ASCs can be delivered in an inject able hydrogel scaffold along with angiogenic growth factors to augment soft tissue transfer. due to partial necrosis. To overcome these problems, Yoshimura et al. developed a novel strategy known as Cell-Assisted Lipotransfer (CAL), with concurrent transplantation of aspirated fat and ASCs [62]. Although preliminary results suggested that CAL is superior to conventional lipoinjection (ASC-poor aspirated fat), some degree of fat graft resorption and volume loss is nonetheless unavoidable [63]. Thus, there is still the unfulfilled need to generate de novo adipose tissue constructs that would be indispensable for the translation of adipose tissue engineering into clinical applications.

\section{Wound Healing}

Wound healing is an intricate process that requires the interaction between numerous biological pathways and many different cell types. Repair of cutaneous wounds normally proceeds in three distinct yet overlapping phases: inflammation, cell proliferation, and remodeling [64]. Despite being an orchestrated process, results are highly unpredictable. Problematic and chronic wound management presents challenging clinical scenarios. Even under optimal conditions, and with current best medical and surgical practice, outcomes can be suboptimal with the healing process resulting in scarring and fibrosis.

Diabetes and burns injuries have served as prototype models for problematic wound healing in basic science research. Attempts to promote healing in these challenging milieux have largely focused on increasing systemic or local tissue growth factor levels, with a particular emphasis being placed on promoting angiogenesis at the wound site. However, clinical translation of these approaches have been suboptimal, resulting in a change in the direction of research focus to identify alternative therapies such as bioengineered skin substitutes and stem cell-based therapies.

\section{The seed: cell source}

Stem cells are considered to play a key role in tissue regeneration during wound healing [65]. The mammalian epidermis is organized into Hair Follicles (HF) interspersed with Inter Follicular Epidermis (IFE), which consists of layers of keratinocytes. Epithelial stem cells, located in the HF bulge and the basal layer of the IFE, are thought to have a high capacity for self-renewal, as evidenced by their ability to produce daughter cells that undergo terminal differentiation into keratinocytes [66]. The regeneration of the skin and its appendages is thought to occur by the regulated activation of these dedicated stem cell populations. Stem cells resident in the HF bulge and the basal layer are potential targets for regulating wound healing.

MSCs are an attractive cell type for cell-based therapy to promote dermal regeneration. In addition to their potential to differentiate, they also exhibit substantial trophic support to regenerating tissues[67] While these cells are not members of the epidermal niche, they can affect wound healing and tissue regeneration through many different avenues [68-70]. These cells are capable of migrating to the site of injury or inflammation, and they may stimulate the proliferation and differentiation of different resident progenitor cells, secrete growth factors, participate in remodeling, and modulate the immune and inflammatory response Preclinical studies have demonstrated the ability of MSCs to accelerate wound closure. Using a variety of methods (topical, local and systemic injection, and collagen scaffold) for delivery of stem cells to an excisional wound, numerous studies have demonstrated enhanced cutaneous wound repair with MSCs in both wild-type and diabetic mice [71-76]. Falanga et al. [77] showed the prevention of ulceration and acceleration of wound closure in mice by spraying autologous BM-MSCs with a mixture of fibrin and thrombin into wounds. The same method applied in a small human clinical trial 
proved to accelerate wound closure and resurfacing without adverse effects [77]. Additionally, there was a strong correlation between the number of cells and the reduction of ulcer area when applied to chronic wounds [77]. Another small clinical trial examined the topical application of culture expanded autologous BM-MSCs on chronic ulcers unresponsive to prior treatment and were able to achieve wound closure with increased cellularity and dermal rebuilding [78]. Although these studies show great promise, larger clinical trials as well as less invasive harvesting techniques are necessary before this can become a more practical therapy (Figure 4).

In juxtaposition, ASCs secrete nearly all the growth factors involved in wound healing and have been shown to remain viable at the wound site [79-81]. Utilizing an excisional wound healing mouse model, Kim et al. delivered a collagen gel solution with ASCs and found a significant reduction of wound size with acceleration of reepithelialization [81]. In another study, human a cellular dermal matrix seeded with ASCs also resulted in acceleration of wound closure [82]. In addition, differentiation of ASCs into epidermal and endothelial cells as well as dermal fibroblasts was observed [82]. Finally, recent reports have shown the application of ASCs overexpressing vascular endothelial growth factor to accelerate wound healing over unmodified ASCs [83]. These results thus attest to the promise of ASCs in cellbased wound regeneration.

\section{The soil: dermal stem cell niche}

In wound repair, the goal is to transiently increase expression of specific growth factors to improve angiogenesis, increase cell proliferation and improve cell migration until complete wound closure is achieved. The interactions between the mesenchymal environments in which epithelial stem cells reside are currently being investigated, with the aim of defining specific molecular regulators that govern stem cell behavior and ultimately determines their fate. Current therapies are only partially effective because they do not inhibit the cell and extracellular matrix proliferation that leads to hypertrophic scar formation. Using matrices seeded with stem cells from the bone marrow, adipose tissue, or hair follicles, development of a fully functional skin substitute that could assure its revascularization, reinnervation, and replacement of skin appendages are potential translational opportunities for the development of novel woundhealing therapies, some of which are already used in clinical practice.

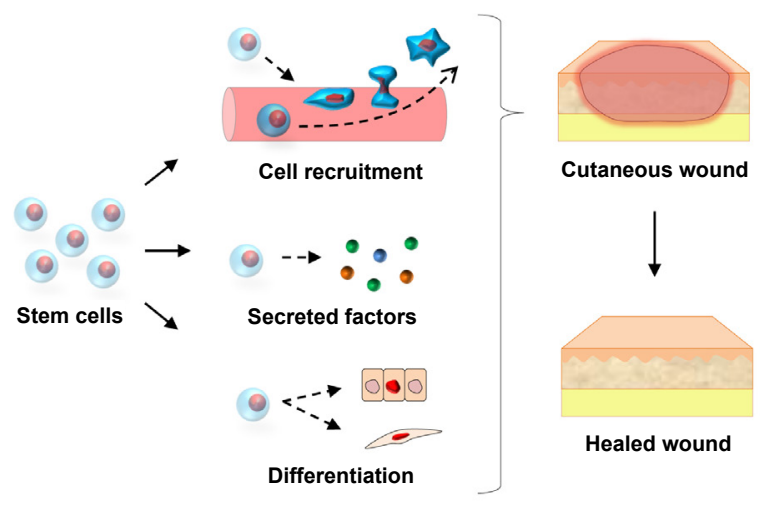

Figure 4: BM-MSCs play a key role in dermal wound healing by either directly replenishing the injured tissue via differentiation, or by recruiting other cells to aid in repair.

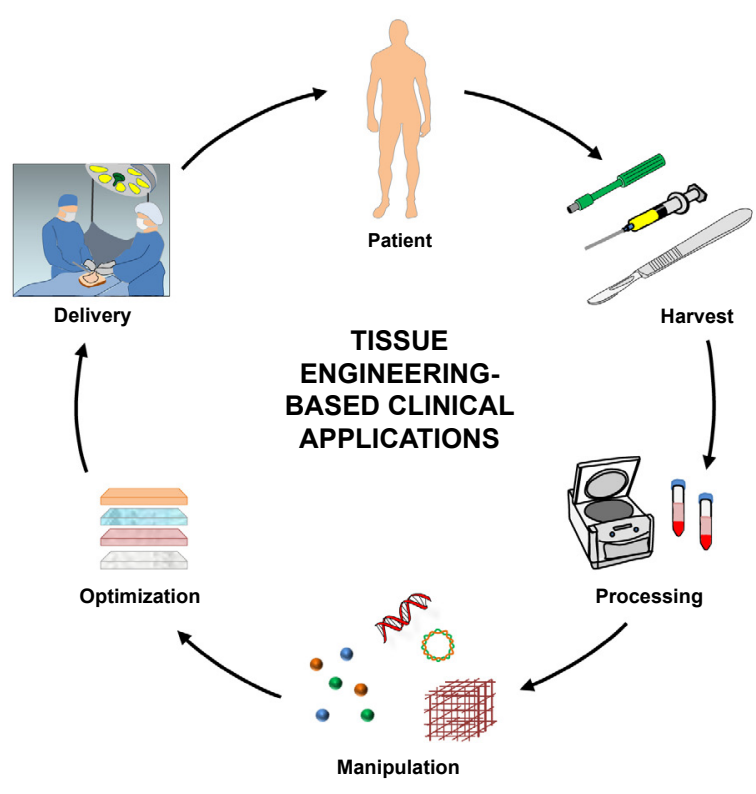

Figure 5: Freshly harvested stem cells may one day be enriched and combined with growth factors and biomimetic scaffolds for clinical tissue engineering applications.

\section{Surgical applications for dermal wound tissue engineering}

The promotion of wound healing using dermal substitutes has become increasingly widespread, but the outcomes of substituteassisted healing remain poor. Despite the wide variety of skin substitutes currently on the market, they are fraught with problems including poor integration with host tissue, relatively high infection rates, scar formation and wound contraction.

The most successful skin substitutes currently available a cellular polymer matrices which are designed to mimic the dermal extracellular matrix and promote a bottom-up approach to wound regeneration. These matrices rely upon a three-dimensional scaffold which is analogous to the extracellular matrix, to can guide cell adhesion, growth and differentiation to form skin function and structural tissue [84].

The application of scaffolds containing viable cells has proven to be problematic due to short shelf-life, high cost and death of transplanted cells as a result of immune rejection and apoptosis [85]. Efforts to increase the effectiveness of skin substitutes employ recent advances in biomaterial science, like bioactivation, which expands their role from being a simple structure to a delivery vehicle for growth factors, cytokines and genes, thus actively encouraging tissue regeneration [86].

\section{Conclusion}

Stem cells and their ability to restore multiple tissue types offer a potentially powerful therapeutic approach for regenerative medicine. While much progress has been made in developing preliminary strategies for clinical applications of stem cells, a deeper understanding of the fundamental mechanisms governing stem cell survival, proliferation, and tissue-directed differentiation, both in isolation and in the complex environments of a heterogeneous array of donor sites, will be required in order to optimally manipulate cells and their microenvironments for widespread patient use. Continued work in our own laboratory and others focus on understanding specific biochemical pathways crucial to the survival and differentiation trajectories of various stem cell classes. 
Citation: McArdle A, Paik KJ, Chung MT, Hu MS, Walmsley G, et al. (2013) Manipulation of Stem Cells and their Microenvironment for Tissue Engineering. Surgery Curr Res 3: 134. doi:10.4172/2161-1076.1000134

Page 6 of 8

Simultaneously, investigations are being performed on manipulating external signaling cues in the form of small molecules, nanoparticles, proteins, or nucleic acids embedded within a variety of biologicallyderived and biomimetic synthetic scaffold environments.

Additional challenges still remain for use of stem cells in therapeutic strategies. These include the need for amplifying cell numbers and optimizing delivery into human patients while avoiding immunogenicity, massive cell death, or tumor formation. And with patient-specific therapies, hurdles must still be overcome with efficient reprogramming and gene modification on a clinically useful time scale. As stem cell therapies progress through clinical trials and into more widespread use, care must be taken to ensure their efficacy and safety in human patients, a process which will likely involve the manipulation of cell-intrinsic factors as well as the niche microenvironment and the ultimate graft site. Nonetheless, with increasing experience and expertise in various stem cells, expanded use of these powerful building blocks for tissue engineering applications in many regenerative medicine specialties will undoubtedly follow.

\section{References}

1. Shang Q, Wang Z, Liu W, Shi Y, Cui L, et al. (2001) Tissue-engineered bone repair of sheep cranial defects with autologous bone marrow stromal cells. J Craniofac Surg 12: 586-593.

2. Kruyt MC, Dhert WJ, Yuan H, Wilson CE, van Blitterswijk CA, et al. (2004) Bone tissue engineering in a critical size defect compared to ectopic implantations in the goat. J Orthop Res 22: 544-551.

3. Kon E, Muraglia A, Corsi A, Bianco P, Marcacci M, et al. (2000) Autologous bone marrow stromal cells loaded onto porous hydroxyapatite ceramic accelerate bone repair in critical-size defects of sheep long bones. Journal of biomedical materials research 49:328-337.

4. Schimming R, Schmelzeisen R (2004) Tissue-engineered bone for maxillary sinus augmentation. J Oral Maxillofac Surg 62: 724-729.

5. Quarto R, Mastrogiacomo M, Cancedda R, Kutepov SM, Mukhachev V, et al (2001) Repair of large bone defects with the use of autologous bone marrow stromal cells. N Engl J Med 344: 385-386.

6. Pittenger MF, Mackay AM, Beck SC, Jaiswal RK, Douglas R, et al. (1999) Multilineage potential of adult human mesenchymal stem cells. Science 284 143-147.

7. Lee HJ, Choi BH, Min BH, Park SR (2009) Changes in surface markers of human mesenchymal stem cells during the chondrogenic differentiation and dedifferentiation processes in vitro. Arthritis Rheum 60: 2325-2332.

8. Petrie Aronin CE, Tuan RS (2010) Therapeutic potential of the immunomodulatory activities of adult mesenchymal stem cells. Birth Defects Res C Embryo Today 90: 67-74.

9. Kolf CM, Cho E, Tuan RS (2007) Mesenchymal stromal cells. Biology of adult mesenchymal stem cells: regulation of niche, self-renewal and differentiation. Arthritis Res Ther 9: 204.

10. Zhao Z, Zhao M, Xiao G, Franceschi RT (2005) Gene transfer of the Runx2 transcription factor enhances osteogenic activity of bone marrow stromal cells in vitro and in vivo. Mol Ther 12: 247-253.

11. Ito T, Sawada R, Fujiwara Y, Tsuchiya T (2008) FGF-2 increases osteogenic and chondrogenic differentiation potentials of human mesenchymal stem cells by inactivation of TGF-beta signaling. Cytotechnology 56: 1-7.

12. Ono M, Inkson CA, Kilts TM, Young MF (2011) WISP-1/CCN4 regulates osteogenesis by enhancing BMP-2 activity. J Bone Miner Res 26: 193-208.

13. D'Ippolito G, Diabira, S Howard, G.A Menei, P Roos, et al. (2004) Marrowisolated adult multilineage inducible (MIAMI) cells, a unique population of postnatal young and old human cells with extensive expansion and differentiation potential. J Cell Sci 117: 2971-2981.

14. Bianchi G, Banfi A, Mastrogiacomo M, Notaro R, Luzzatto L, et al. (2003) Ex vivo enrichment of mesenchymal cell progenitors by fibroblast growth factor 2 . Exp Cell Res 287: 98-105.
15. Carrancio S, López-Holgado N, Sánchez-Guijo FM, Villarón E, Barbado V, et al. (2008) Optimization of mesenchymal stem cell expansion procedures by cell separation and culture conditions modification. Exp Hematol 36: 1014-1021.

16. Takahashi K, Yamanaka S (2006) Induction of pluripotent stem cells from mouse embryonic and adult fibroblast cultures by defined factors. Cell 126 663-676.

17. Sottile V, Thomson A, McWhir J (2003) In vitro osteogenic differentiation of human ES cells. Cloning Stem Cells 5: 149-155.

18. Kawaguchi J, Mee PJ, Smith AG (2005) Osteogenic and chondrogenic differentiation of embryonic stem cells in response to specific growth factors. Bone 36: 758-769.

19. Tashiro K, Inamura M, Kawabata K, Sakurai F, Yamanishi K, et al. (2009) Efficient adipocyte and osteoblast differentiation from mouse induced pluripotent stem cells by adenoviral transduction. Stem Cells 27: 1802-1811.

20. Li F, Bronson S, Niyibizi C (2010) Derivation of murine induced pluripotent stem cells (iPS) and assessment of their differentiation toward osteogenic lineage. $J$ Cell Biochem 109: 643-652.

21. Lian Q, Zhang Y, Zhang J, Zhang HK, Wu X, et al. (2010) Functional mesenchymal stem cells derived from human induced pluripotent stem cells attenuate limb ischemia in mice. Circulation 121: 1113-1123.

22. Levi B, Hyun JS, Montoro DT, Lo DD, Chan CK, et al. (2012) In vivo directed differentiation of pluripotent stem cells for skeletal regeneration. Proc Natl Acad Sci U S A 109: 20379-20384.

23. Duan X, Tu Q, Zhang J, Ye J, Sommer C, et al. (2011) Application of induced pluripotent stem (iPS) cells in periodontal tissue regeneration. J Cell Physio 226: $150-157$

24. Young S, Wong M, Tabata Y, Mikos AG (2005) Gelatin as a delivery vehicle for the controlled release of bioactive molecules. J Control Release 109: 256-274.

25. Kim J, Kim IS, Cho TH, Lee KB, Hwang SJ, et al. (2007) Bone regeneration using hyaluronic acid-based hydrogel with bone morphogenic protein-2 and human mesenchymal stem cells. Biomaterials 28: 1830-1837.

26. Reichert JC, Heymer A, Berner A, Eulert J, Nöth U (2009) Fabrication of polycaprolactone collagen hydrogel constructs seeded with mesenchyma stem cells for bone regeneration. Biomed Mater 4: 065001.

27. Zhao J, Shinkai M, Takezawa T, Ohba S, Chung UI, et al. (2009) Bone regeneration using collagen type I vitrigel with bone morphogenetic protein-2. J Biosci Bioeng 107: 318-323.

28. Santo VE, Gomes ME, Mano JF, Reis RL (2012) Chitosan-chondroitin sulphate nanoparticles for controlled delivery of platelet lysates in bone regenerative medicine. J Tissue Eng Regen Med 6 Suppl 3: s47-59.

29. Kellomäki M, Niiranen H, Puumanen K, Ashammakhi N, Waris T, et al (2000) Bioabsorbable scaffolds for guided bone regeneration and generation Biomaterials 21: 2495-2505.

30. Di Bella C, Farlie P, Penington AJ (2008) Bone regeneration in a rabbit criticalsized skull defect using autologous adipose-derived cells. Tissue engineering Part A 14: 483-490.

31. Rezwan K, Chen QZ, Blaker JJ, Boccaccini AR (2006) Biodegradable and bioactive porous polymer/inorganic composite scaffolds for bone tissue engineering. Biomaterials 27: 3413-3431.

32. Kawate K, Yajima H, Ohgushi H, Kotobuki N, Sugimoto K, et al. (2006) Tissueengineered approach for the treatment of steroid-induced osteonecrosis of the femoral head: transplantation of autologous mesenchymal stem cells cultured with beta-tricalcium phosphate ceramics and free vascularized fibula. Artificia organs 30: 960-962.

33. Woodard JR, Hilldore AJ, Lan SK, Park CJ, Morgan AW, et al. (2007) The mechanical properties and osteoconductivity of hydroxyapatite bone scaffolds with multi-scale porosity. Biomaterials 28: 45-54

34. Fielding GA, Bandyopadhyay A, Bose S (2012). Effects of silica and zinc oxide doping on mechanical and biological properties of 3D printed tricalcium phosphate tissue engineering scaffolds. Dental materials : official publication of the Academy of Dental Materials 28: 113-122.

35. Mankani MH, Krebsbach PH, Satomura K, Kuznetsov SA, Hoyt R, et al. (2001) Pedicled bone flap formation using transplanted bone marrow stromal cells. Arch Surg 136: 263-270. 
Citation: McArdle A, Paik KJ, Chung MT, Hu MS, Walmsley G, et al. (2013) Manipulation of Stem Cells and their Microenvironment for Tissue Engineering. Surgery Curr Res 3: 134. doi:10.4172/2161-1076.1000134

36. Warnke PH, Springer IN, Wiltfang J, Acil Y, Eufinger H, et al. (2004) Growth and transplantation of a custom vascularised bone graft in a man. Lancet 364 : 766-770

37. Katz AJ, Llull R, Hedrick MH, Futrell JW (1999) Emerging approaches to the tissue engineering of fat. Clin Plast Surg 26: 587-603, viii.

38. Patrick CW Jr (2001) Tissue engineering strategies for adipose tissue repair Anat Rec 263: 361-366.

39. Brey EM, Patrick CW Jr (2000) Tissue engineering applied to reconstructive surgery. IEEE Eng Med Biol Mag 19: 122-125.

40. Halberstadt C, Austin C, Rowley J, Culberson C, Loebsack A, et al. (2002) A hydrogel material for plastic and reconstructive applications injected into the subcutaneous space of a sheep. Tissue Eng 8: 309-319.

41. Fischbach C, Spruss T, Weiser B, Neubauer M, Becker C, et al. (2004) Generation of mature fat pads in vitro and in vivo utilizing 3-D long-term culture of 3T3-L1 preadipocytes. Exp Cell Res 300: 54-64.

42. Fischbach C, Seufert J, Staiger H, Hacker M, Neubauer M, et al. (2004) Threedimensional in vitro model of adipogenesis: comparison of culture conditions. Tissue Eng 10: 215-229.

43. Alhadlaq A, Tang M, Mao JJ (2005) Engineered adipose tissue from human mesenchymal stem cells maintains predefined shape and dimension: implications in soft tissue augmentation and reconstruction. Tissue Eng 11 556-566.

44. Green H, Kehinde O (1976) Spontaneous heritable changes leading to increased adipose conversion in 3T3 cells. Cell 7: 105-113.

45. Russell TR, Ho R (1976) Conversion of 3 T3 fibroblasts into adipose cells: triggering of differentiation by prostaglandin F2alpha and 1-methyl-3-isobutyl xanthine. Proc Natl Acad Sci U S A 73: 4516-4520.

46. Fernyhough ME, Vierck JL, Hausman GJ, Mir PS, Okine EK, et al. (2004) Primary adipocyte culture: adipocyte purification methods may lead to a new understanding of adipose tissue growth and development. Cytotechnology 46 163-172.

47. Fernyhough ME, Bucci LR, Hausman GJ, Antonio J, Vierck JL, et al. (2005) Gaining a solid grip on adipogenesis. Tissue Cell 37: 335-338.

48. Zuk PA, Zhu M, Ashjian P, De Ugarte DA, Huang Jl, et al. (2002) Human adipose tissue is a source of multipotent stem cells. Mol Biol Cell 13: 4279-

49. von Heimburg D, Zachariah S, Heschel I, Kühling H, Schoof $H$, et al. (2001) Human preadipocytes seeded on freeze-dried collagen scaffolds investigated in vitro and in vivo. Biomaterials 22: 429-438.

50. Locke M, Feisst V, Dunbar PR (2011) Concise review: human adipose-derived stem cells: separating promise from clinical need. Stem Cells 29: 404-411.

51. Li H, Zimmerlin L, Marra KG, Donnenberg VS, Donnenberg AD, et al. (2011) Adipogenic potential of adipose stem cell subpopulations. Plast Reconstr Surg 128: $663-672$.

52. Kawaguchi N, Toriyama K, Nicodemou-Lena E, Inou K, Torii S, et al. (1998) De novo adipogenesis in mice at the site of injection of basement membrane and basic fibroblast growth factor. Proc Natl Acad Sci U S A 95: 1062-1066.

53. Kimura Y, Ozeki M, Inamoto T, Tabata $Y$ (2002) Time course of de novo adipogenesis in matrigel by gelatin microspheres incorporating basic fibroblas growth factor. Tissue Eng 8: 603-613.

54. Masuda T, Furue M, Matsuda T (2004). Photocured, styrenated gelatin-based microspheres for de novo adipogenesis through corelease of basic fibroblas growth factor, insulin, and insulin-like growth factor I. Tissue engineering 10 $523-535$

55. Toriyama K, Kawaguchi N, Kitoh J, Tajima R, Inou K, et al. (2002) Endogenous adipocyte precursor cells for regenerative soft-tissue engineering. Tissue Eng 8: 157-165.

56. Croissandeau G, Basak A, Seidah NG, Chrétien M, Mbikay M (2002) Proprotein convertases are important mediators of the adipocyte differentiation of mouse 3T3-L1 cells. J Cell Sci 115: 1203-1211.

57. Mandrup S, Lane MD (1997) Regulating adipogenesis. J Biol Chem 272: 5367 5370

58. Yuksel E, Weinfeld AB, Cleek R, Waugh JM, Jensen J, et al. (2000) De novo adipose tissue generation through long-term, local delivery of insulin and insulin-like growth factor-1 by PLGA/PEG microspheres in an in vivo rat model: a novel concept and capability. Plast Reconstr Surg 105: 1721-1729.

59. Flynn L, Woodhouse KA (2008) Adipose tissue engineering with cells in engineered matrices. Organogenesis 4: 228-235.

60. Patrick CW Jr, Zheng B, Johnston C, Reece GP (2002) Long-term implantation of preadipocyte-seeded PLGA scaffolds. Tissue Eng 8: 283-293.

61. Kim WS, Mooney DJ, Arany PR, Lee K, Huebsch N, et al. (2012) Adipose tissue engineering using injectable, oxidized alginate hydrogels. Tissue Eng Part A 18: 737-743.

62. Matsumoto D, Sato K, Gonda K, Takaki Y, Shigeura T, et al. (2006) Cellassisted lipotransfer: supportive use of human adipose-derived cells for soft tissue augmentation with lipoinjection. Tissue Eng 12: 3375-3382.

63. Yoshimura K, Sato K, Aoi N, Kurita M, Hirohi T, et al. (2008) Cell-assisted lipotransfer for cosmetic breast augmentation: supportive use of adiposederived stem/stromal cells. Aesthetic Plast Surg 32: 48-55

64. Singer AJ, Clark RA (1999) Cutaneous wound healing. N Engl J Med 341 738-746.

65. Lau K, Paus R, Tiede S, Day P, Bayat A (2009) Exploring the role of stem cells in cutaneous wound healing. Exp Dermatol 18: 921-933.

66. Watt FM (1998) Epidermal stem cells: markers, patterning and the control of stem cell fate. Philos Trans R Soc Lond B Biol Sci 353: 831-837.

67. Jackson WM, Nesti LJ, Tuan RS (2012) Concise review: clinical translation of wound healing therapies based on mesenchymal stem cells. Stem Cells Trans Med 1: 44-50.

68. Chamberlain G, Fox J, Ashton B, Middleton J (2007) Concise review: mesenchymal stem cells: their phenotype, differentiation capacity, immunological features, and potential for homing. Stem Cells 25: 2739-2749.

69. Caplan Al (2007) Adult mesenchymal stem cells for tissue engineering versus regenerative medicine. J Cell Physiol 213: 341-347.

70. Uccelli A, Pistoia V, Moretta L (2007) Mesenchymal stem cells: a new strategy for immunosuppression? Trends Immunol 28: 219-226.

71. Wu Y, Chen L, Scott PG, Tredget EE (2007) Mesenchymal stem cells enhance wound healing through differentiation and angiogenesis. Stem Cells 25: 2648 2659 .

72. Chen L, Tredget EE, Wu PY, Wu Y (2008) Paracrine factors of mesenchyma stem cells recruit macrophages and endothelial lineage cells and enhance wound healing. PLoS One 3: e1886.

73. Chen L, Tredget EE, Liu C, Wu Y (2009) Analysis of allogenicity of mesenchymal stem cells in engraftment and wound healing in mice. PLoS One 4: e7119.

74. Sasaki M, Abe R, Fujita Y, Ando S, Inokuma D, et al. (2008) Mesenchyma stem cells are recruited into wounded skin and contribute to wound repair by transdifferentiation into multiple skin cell type. J Immunol 180: 2581-2587.

75. Egaña JT, Fierro FA, Krüger S, Bornhäuser M, Huss R, et al. (2009) Use of human mesenchymal cells to improve vascularization in a mouse model for scaffold-based dermal regeneration. Tissue Eng Part A 15: 1191-1200.

76. Javazon EH, Keswani SG, Badillo AT, Crombleholme TM, Zoltick, et al. (2007) Enhanced epithelial gap closure and increased angiogenesis in wounds of diabetic mice treated with adult murine bone marrow stromal progenitor cells Wound repair and regeneration:official publication of the Wound Healing Society [and] the European Tissue Repair Society 15: 350-359.

77. Falanga V, Iwamoto S, Chartier M, Yufit T, Butmarc J, et al. (2007) Autologous bone marrow-derived cultured mesenchymal stem cells delivered in a fibrin spray accelerate healing in murine and human cutaneous wounds. Tissue Eng 13: $1299-1312$

78. Badiavas EV, Falanga V (2003) Treatment of chronic wounds with bone marrow-derived cells. Arch Dermatol 139: 510-516.

79. Ebrahimian TG, Pouzoulet F, Squiban C, Buard V, Andre, et al. (2009) Cell therapy based on adipose tissue-derived stromal cells promotes physiological and pathological wound healing. Arteriosclerosis, thrombosis, and vascula biology 29: 503-510.

80. Blanton M W, Hadad I, Johnstone, Mund JA, Rogers, et al. (2009) Adipose stromal cells and platelet-rich plasma therapies synergistically increase revascularization during wound healing. Plastic and reconstructive surgery 123: $56 \mathrm{~S}-64 \mathrm{~S}$. 
Citation: McArdle A, Paik KJ, Chung MT, Hu MS, Walmsley G, et al. (2013) Manipulation of Stem Cells and their Microenvironment for Tissue Engineering. Surgery Curr Res 3: 134. doi:10.4172/2161-1076.1000134

81. Kim WS, Park BS, Sung JH, Yang JM, Park SB, et al. (2007) Wound healing effect of adipose-derived stem cells: a critical role of secretory factors on human dermal fibroblasts. J Dermatol Sci 48: 15-24.

82. Altman AM, Matthias N, Yan Y, Song YH, Bai X, et al. (2008) Dermal matrix as a carrier for in vivo delivery of human adipose-derived stem cells. Biomaterials 29: 1431-1442.

83. Nauta A, Seidel, C Deveza, L Montoro, D Grova, et al. (2013) Adipose-derived stromal cells overexpressing vascular endothelial growth factor accelerate mouse excisional wound healing. Molecular therapy: the journal of the American Society of Gene Therapy 21: 445-455.
84. Zhong SP, Zhang YZ, Lim CT (2010) Tissue scaffolds for skin wound healing and dermal reconstruction. Wiley Interdiscip Rev Nanomed Nanobiotechnol 2 . 510-525.

85. Hodgkinson T, Bayat A (2011) Dermal substitute-assisted healing: enhancing stem cell therapy with novel biomaterial design. Arch Dermatol Res 303: 301 315

86. Yildirimer L, Thanh NT, Seifalian AM (2012) Skin regeneration scaffolds: a multimodal bottom-up approach. Trends Biotechnol 30: 638-648.

This article was originally published in a special issue, Plastic Surgery handled by Editor(s). Dr. Eduardo Krulig, Centro Medico, Spain 